$$
\text { S? }
$$

\title{
Spin mapping of surface and bulk Rashba states in ferroelectric $\alpha$-GeTe(111) films
}

\author{
H. J. Elmers, ${ }^{1,}{ }^{*}$ R. Wallauer, ${ }^{1}$ M. Liebmann, ${ }^{2}$ J. Kellner, ${ }^{2}$ M. Morgenstern, ${ }^{2}$ R. N. Wang, ${ }^{3}$ J. E. Boschker, ${ }^{3}$ R. Calarco, ${ }^{3}$ \\ J. Sánchez-Barriga, ${ }^{4}$ O. Rader, ${ }^{4}$ D. Kutnyakhov, ${ }^{1}$ S. V. Chernov,${ }^{1}$ K. Medjanik, ${ }^{1}$ C. Tusche,${ }^{5,6}$ M. Ellguth, ${ }^{1,5}$ H. Volfova, \\ St. Borek, ${ }^{7}$ J. Braun, ${ }^{7}$ J. Minár, ${ }^{7,8}$ H. Ebert, ${ }^{7}$ and G. Schönhense ${ }^{1}$ \\ ${ }^{1}$ Institut für Physik, Johannes Gutenberg-Universität, Staudingerweg 7, D-55099 Mainz, Germany \\ ${ }^{2}$ II. Physikalisches Institut B and JARA-FIT, RWTH Aachen University, D-52074 Aachen, Germany \\ ${ }^{3}$ Paul-Drude-Institut für Festkörperelektronik, Hausvogteiplatz, 5-7, 10117 Berlin, Germany \\ ${ }^{4}$ Helmholtz-Zentrum Berlin für Materialien und Energie, Elektronenspeicherring BESSY II, \\ Albert-Einstein-Straße 15, D-12489 Berlin, Germany \\ ${ }^{5}$ Max-Planck-Institut für Mikrostruktur Physik, Weinberg 2, D-06120 Halle, Germany \\ ${ }^{6}$ Peter Grünberg Institut (PGI-6), Forschungszentrum Jülich GmbH, 52425 Jülich, Germany \\ ${ }^{7}$ Department Chemie, Ludwig-Maximilians-Universität München, 81377 Munich, Germany \\ ${ }^{8}$ New Technologies-Research Center, University West Bohemia, Univerzitni 8, 30614 Pilseñ, Czech Republic
}

(Received 4 June 2016; revised manuscript received 5 September 2016; published 9 November 2016)

\begin{abstract}
The breaking of bulk inversion symmetry in ferroelectric semiconductors causes a Rashba-type spin splitting of electronic bulk bands. This is shown by a comprehensive mapping of the spin polarization of the electronic bands in ferroelectric $\alpha$-GeTe(111) films using a time-of-flight momentum microscope equipped with an imaging spin filter that enables a simultaneous measurement of more than 10000 data points. The experiment reveals an opposite spin helicity of the inner and outer Rashba bands with a different spin polarization in agreement with theoretical predictions, confirming a complex spin texture of bulk Rashba states. The outer band has about twice larger spin polarization than the inner one, giving evidence of a spin-orbit effect being related to the orbital composition of the band states. The switchable inner electric field of GeTe implies new functionalities for spintronic devices.
\end{abstract}

DOI: 10.1103/PhysRevB.94.201403

The strong coupling of electron momentum and spin in low-dimensional structures permits an electrically controlled spin manipulation in spintronic devices [1-4], e.g., via the Rashba effect [5].

The Rashba effect has first been experimentally demonstrated in semiconductor heterostructures, where an electrical field perpendicular to the layered structure, i.e., perpendicular to the electron momentum, determines the electron spin orientation relative to its momentum [6-8]. An asymmetric interface structure causes the necessary inversion symmetry breaking and accounts for the special spin splitting of electron states, the Rashba effect [5], the size of which can be tuned by the strength of the electrical field.

For most semiconducting materials the Rashba effect generates only a quite small splitting of the order of $10^{-2} \AA^{-1}$. This requires experiments at low temperatures [9-11] and also implies large lateral dimensions for potential spintronic applications. A considerably larger splitting has been predicted theoretically [12] and was recently found experimentally for the surface states of $\operatorname{GeTe}(111)[13,14]$.

GeTe is a ferroelectric semiconductor with a Curie temperature of $700 \mathrm{~K}$. Thus, besides the interface induced Rashba splitting, the ferroelectric properties also imply a broken inversion symmetry within the bulk and, as a consequence would allow for the electrical tuning of the bulk Rashba splitting via switching the ferroelectric polarization $[12,15,16]$. This effect is of great interest for nonvolatile spin orbitronics [10].

For GeTe, a bulk Rashba splitting of $0.19 \AA^{-1}$ has been predicted theoretically [12]. Experimentally, bulk-Rashba

\footnotetext{
*elmers@uni-mainz.de
}

bands are rare and have only been found so far in the layered polar semiconductors BiTeCl and BiTeI [17-21] that, however, are not switchable.

A characterization of the ferroelectric properties and a measurement of the related helical spin polarization of the surface states of $\mathrm{GeTe}(111)$ has been performed previously by force microscopy [22] and spin-resolved angular-resolved photoemission spectroscopy [13], respectively. A combined experimental and theoretical study revealed that at the Fermi level the complex surface electronic structure results in unconventional spin topologies with chiral symmetry [14,23].

Here, we demonstrate the helical spin structure of bulk bands using the GeTe(111) surface but probing states at higher binding energies, which barely interact with surface states. Therefore, we apply spin-resolved time-of-flight momentum microscopy that allows for an effective parallel spin mapping of the electronic states [24]. The technique is based on the significant improvement of spin polarimeters by multichannel detection $[25,26]$. The previous findings for the spin texture of surface states at the Fermi level are confirmed. In addition, we reveal the predicted Rashba-type spin texture of the bulk bands. This is a central precondition for nonvolatile and energy-efficient spin switching memory devices operating independently from surface or interface properties.

Single-domain epitaxial $\alpha-\operatorname{GeTe}(111)$ films [27] with a thickness of $32 \mathrm{~nm}$ are grown by molecular beam epitaxy on in situ prepared $\mathrm{Si}(111)$ and are transferred in ultrahigh vacuum to the spin-resolved time-of-flight momentum microscope. Thus, we are able to probe the pristine surface, which shows an outward ferroelectric polarization. It was previously shown that a metastable inverted polarization state can be written using ex situ piezoforce microscopy. Details of the structural 
and ferroelectric properties of the samples are reported in Ref. [13].

The photoemission experiment has been performed at the undulator beamline U125-NIM at BESSY II in the single bunch mode, providing vacuum ultraviolet photon pulses (photon energy range $15-27 \mathrm{eV}$ ) of 50 ps duration and a repetition rate of $1.25 \mathrm{MHz}$. The time-of-flight momentum microscope yields a simultaneous acquisition of the $I\left(E_{b}, k_{x}, k_{y}\right)$ spectral function in the full surface Brillouin zone and several eV energy interval. $E_{b}=E_{F}-E$ denotes the binding energy and $k_{x}, k_{y}$ the electron momentum parallel to the surface. Details of the instrument are given in Ref. [24]. For the present experiment the overall energy and the $k_{\|}$resolution are $86 \mathrm{meV}$ and $0.04 \AA^{-1}$. The linearly polarized photon beam illuminates the sample at an angle of incidence of $68^{\circ}$ with respect to the surface normal and parallel to the $\bar{\Gamma}-\bar{M}$ direction with the electric field vector parallel to the plane of incidence ( $p$ polarization). Spin detection was achieved by electron reflection at the $\mathrm{W}(100)$ spin filter crystal in the [010] azimuth at $45^{\circ}$ reflection angle $[25,28]$. The spin direction parallel to the $\bar{\Gamma}-\bar{M}$ direction is probed. Two datasets $I^{\sigma}\left(E, k_{\|}\right), \sigma=1,2$, were acquired at scattering energies 26.5 and $30.5 \mathrm{eV}$, respectively. At $26.5 \mathrm{eV}$ the reflection asymmetry is $A=0.42$ [28], while it is negligibly small at $30.5 \mathrm{eV}$. The spin polarization is then determined by

$$
P\left(E, k_{\|}\right)=\frac{2}{A} \frac{I^{1}\left(E, k_{\|}\right)-r(E) I^{2}\left(E, k_{\|}\right)}{I^{1}\left(E, k_{\|}\right)+r(E) I^{2}\left(E, k_{\|}\right)},
$$

where $r(E)$ denotes the energy-dependent relative reflection coefficient as described in Ref. [29]. The energy dependence of $A$ was neglected as we analyzed only a narrow energy interval of less than $1 \mathrm{eV}$.

Photoemission calculations are performed based on $a b$ initio ground state properties of $\mathrm{GeTe}(111)$. The calculations were done by a fully relativistic one-step model in its spin-density matrix formulation as implemented in the spin-polarized relativistic Korringa-Kohn-Rostoker package [30,31]. This approach allows describing properly the complete spinpolarization vector in particular for Rashba systems using spin-polarized low-energy electron diffraction theory for the construction of the initial and final states. More details of the computational method are described in Ref. [32].

First, we describe spin-integrated experimental results. Figure 1(a) shows a sketch of the surface and bulk first Brillouin zone of GeTe(111). The known sixfold symmetric shape of the bulk Rashba band [12] is displayed on the hexagonal surface normal to $k_{z}$ of the bulk Brillouin zone defined by the symmetry points $Z A U$ [see Fig. 1(a)]. Figure 1(b) presents a constant energy slice at the Fermi level $E=E_{F}$ covering the complete first bulk Brillouin zone measured with a photon energy of $18.5 \mathrm{eV}$. According to Ref. [13] $h v=18.5 \mathrm{eV}$ probes the bulk bands at $E_{F}$ in the vicinity of the $Z A U$ plane.

Figure 1(c) shows a magnified energy isosurface at a binding energy $E_{b}=0.4 \mathrm{eV}$ measured at a photon energy of $h v=22 \mathrm{eV}$. The split surface Rashba bands with sixfold symmetry are clearly resolved. Additional arcs marked $B_{1}$ appear at lower $|k|$ revealing a threefold symmetry. In order to prove that these arcs are bulk bands we performed the experiment for a number of photon energies from $h v=15.5 \mathrm{eV}$ to (a)
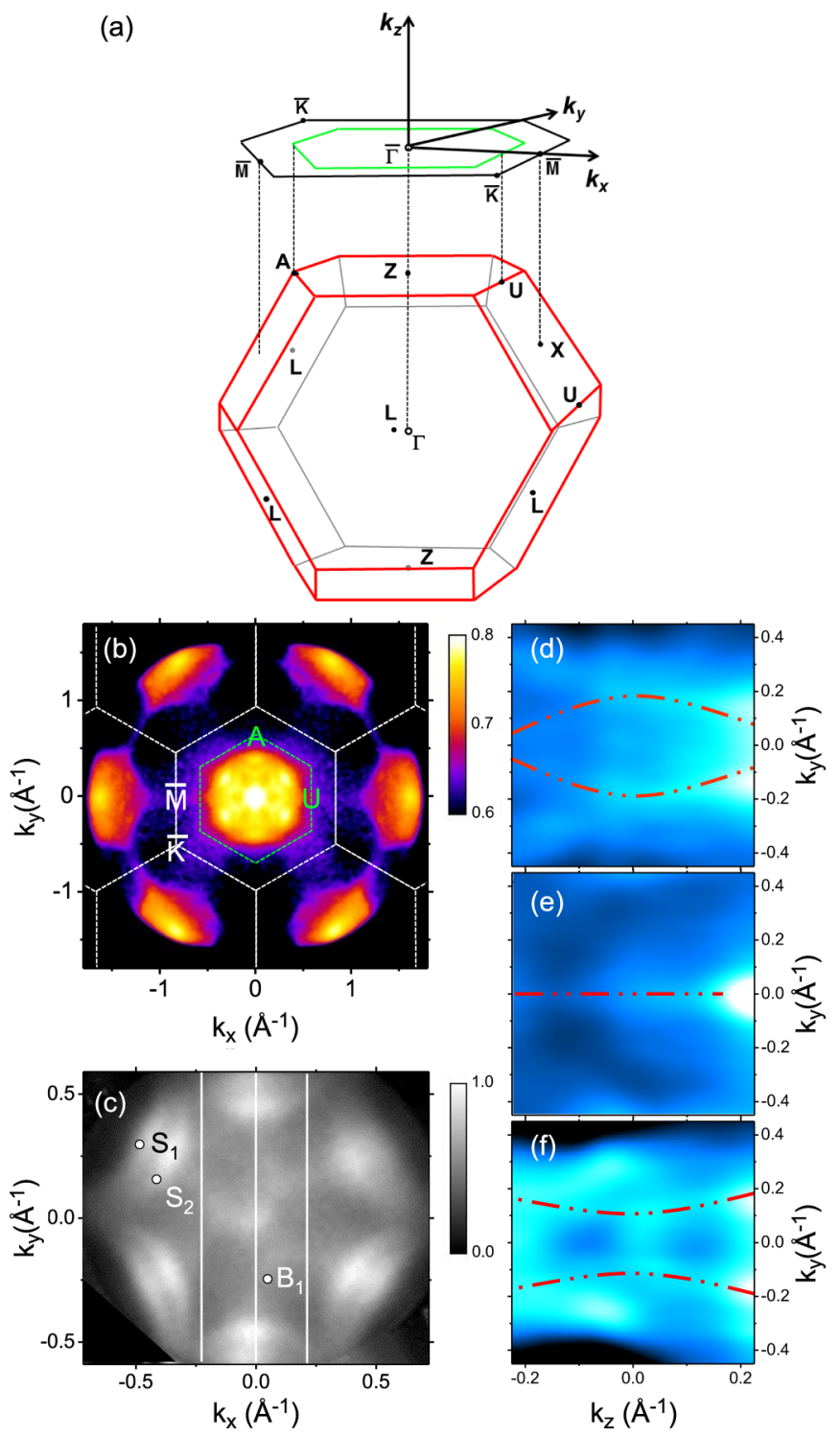

FIG. 1. (a) Bulk and surface Brillouin zone of GeTe with marked high symmetry points and the projection of the hexagonal face of the Brillouin zone encircled in green. (b) Constant energy slice $I\left(E_{F}, k_{x}, k_{y}\right)$ of the photoelectron intensity distribution for a photon energy of $h v=18.5 \mathrm{eV}$ covering the complete bulk Brillouin zone projection. (c) Constant energy slice $I\left(E_{b}, k_{x}, k_{y}\right)$ with $E_{b}=0.4 \mathrm{eV}$ for $h v=22 \mathrm{eV}$ with larger magnification. The two surface bands $\left(S_{1,2}\right)$ and the bulk band $B_{1}$ are marked. Full lines at constant $k_{x}$ indicate positions of cuts shown in (d)-(f). (d)-(f) Photoelectron intensity $I\left(0.4 \mathrm{eV}, k_{x}, k_{y}, k_{z}\right)$ for fixed values $k_{x}=0$ and $k_{x}= \pm 0.2 \AA^{-1} \cdot k_{z}$ is measured relative to the $Z$ point in $k$ space, which is probed for $h v=19.5 \mathrm{eV}$. Dash-dotted lines indicate bulk bands.

$h v=24 \mathrm{eV}$ in steps of $0.5 \mathrm{eV}$ resulting in the four-dimensional dataset $I\left(E_{b}, k_{x}, k_{y}, k_{z}\right)$. Each of the $k_{z}=$ const subsets took about $15 \mathrm{~min}$ of acquisition time. From the data we extracted constant energy maps $I\left(0.4 \mathrm{eV}, k_{x}, k_{y}, k_{z}\right)$ at a binding energy $E_{b}=0.4 \mathrm{eV}$ with fixed values $k_{x}=0$ and $k_{x}= \pm 0.2 \AA^{-1}$ [Figs. 1(d)-1(f)]. Bulk bands are identified by their dispersion with $k_{z}$ [see Figs. 1(d) and 1(f)]. The symmetric behavior of the 
dispersion allows the conclusion that the high symmetry point corresponds to a photon energy of $19.5 \mathrm{eV}$. Using the known radius of the Brillouin zone $\Gamma Z=0.91 \AA^{-1}$ and assuming an excitation into free electron states, we derive an inner potential of $E_{F}-E_{i}=9.2 \mathrm{eV}$, agreeing well with theoretical values [13]. In contrast, the dispersionless behavior of the band shown in Fig. 1(e) indicates its nature as a surface resonant band.

In the following, we discuss the spin polarization of the electronic bands measured after reflection at the W(100) spin filter crystal probing the $x$ component $P_{x}$. In order to remove artificial experimental asymmetries we exploit the fact that the $(\Gamma, Z, U)$ plane represents a mirror plane of the whole experiment including the illumination geometry and polarization. Hence, the spin polarization has been symmetrized in accordance with $P_{x}\left(E_{b}, k_{x}, k_{y}\right)=-P_{x}\left(E_{b}, k_{x},-k_{y}\right)$. From the complete three-dimensional $P_{x}\left(E_{b}, k_{x}, k_{y}\right)$ array we present constant energy slices at selected binding energies in Figs. 2(a)2(g). Each slice integrates over an energy interval of 43 meV. At $E_{F}$ [Fig. 2(a)] the spin vector is oriented clockwise (counterclockwise) for the outer (inner) surface Rashba band SR seen from above the sample surface. The maximum value $P_{x}=0.7 \pm 0.1$ is observed at the top and bottom of the outer Rashba surface band. The left and right outer Rashba surface bands show a reduced polarization of $P_{x}=0.35 \pm$ 0.1 in rough agreement with the Rashba-type spin texture, i.e., $\vec{P} \perp \vec{k}_{\|}$(see a detailed comparison in Ref. [33]). Spin polarizations for the inner bands are of opposite direction and only slightly smaller. These results confirm previously published data $[13,14]$.

The energy isosurface at $E-E_{F}=-0.565 \mathrm{eV}$ shown in Fig. 2(f) also reveals the spin orientation of the bulk Rashba band BR which is crucial because the Rashba splitting is not resolved in the intensity signal. In order to emphasize the weak spin contrast we present the $E\left(k_{y}\right)$ cuts in Figs. 3(a) and 3(b) parallel to $Z A$ for $k_{x}=0$ and $k_{x}=0.09 \AA^{-1}$, the latter representing a cut through the bulk band. The spin direction is essentially perpendicular to the $E\left(k_{y}\right)$ cut shown in Fig. 3(b) and it is of opposite sign to the left and right from the bulk band's maximum intensity. The order of spin directions, moreover, inverts for the bands on the left and on the right of the center, i.e., the spin texture is helical. The $P_{x}$ component for the larger absolute $k_{\|}$of the Rashba split band is also parallel to that of the surface band with larger $k_{\|}$value, which is in agreement with the theoretical predictions of the Rashba-type spin orientation for the equilibrium ferroelectric polarization of GeTe $[13,14]$.

Considering the excitation geometry of the experiment, the photoemission calculations reveal the initial state polarization except for the absolute value of the spin polarization [see Figs. 3(c) and 3(d)]. The $E\left(k_{x}=0, k_{y}\right)$ cut in Fig. 3(c) shows the spin polarization of the Rashba surface states SR crossing the Fermi level at $k_{y}=0.5 \AA^{-1}$ and at $k_{y}=0.1 \AA^{-1}$. Near the Fermi level, bulk states strongly hybridize with surface states forming surface resonances [see arrows in Figs. 2(b) and 2(d)] [14]. In contrast bulk Rashba states BR are separated from surface states SR at a binding energy larger than $0.4 \mathrm{eV}$. Here, their bulk Rashba splitting can be definitely identified. The theoretical $E\left(k_{x}=0.1 \AA^{-1}, k_{y}\right)$ cut in Fig. 3(d) shows the
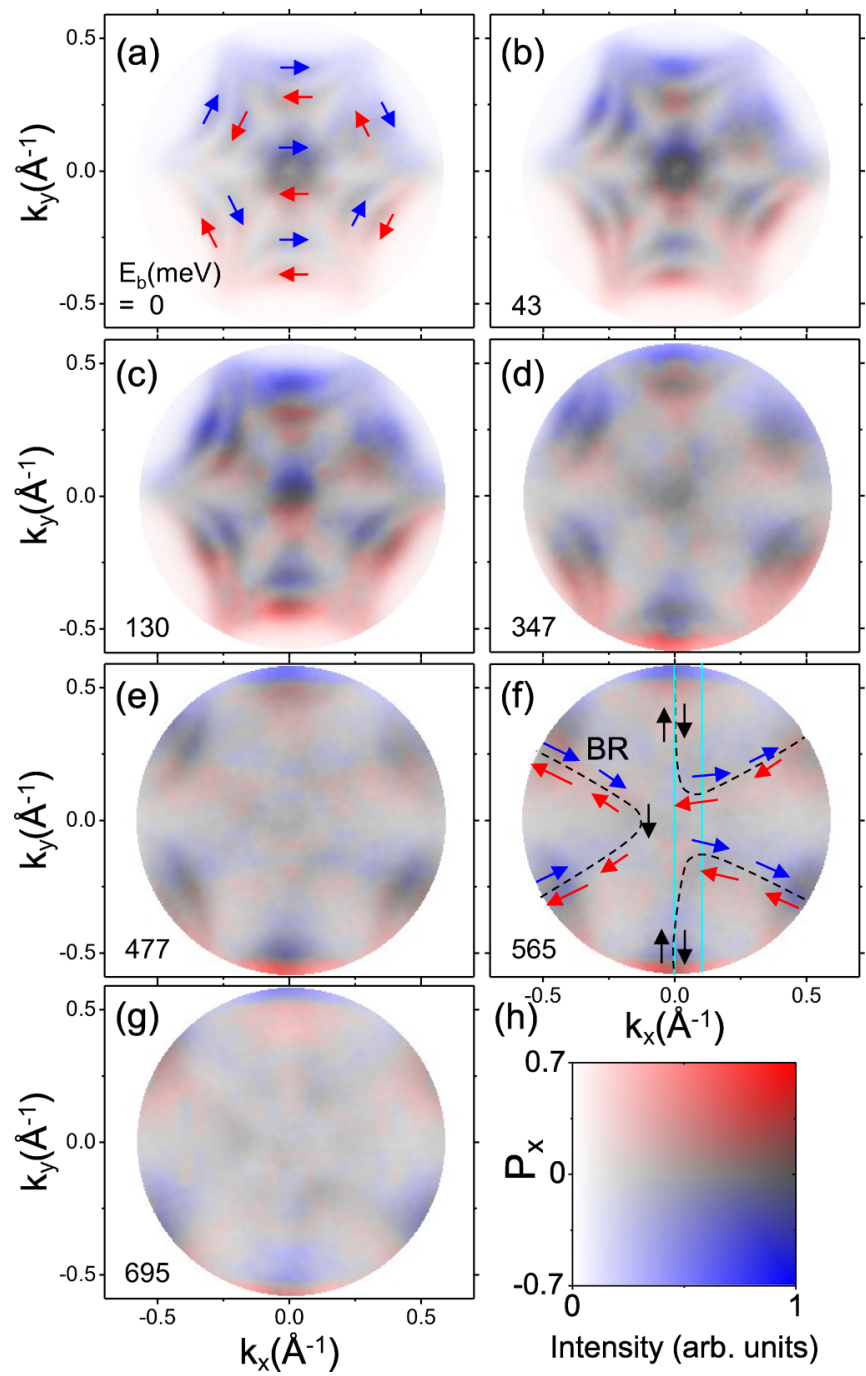

FIG. 2. (a)-(g) Energy isosurfaces of the photoelectron spinpolarization component $P_{x}\left(E_{b}, k_{x}, k_{y}\right)$ combined with the corresponding intensity distribution measured without spin resolution for $h v=22 \mathrm{eV}$. Each slice averages over an energy interval of $43 \mathrm{meV}$. The corresponding color code for $P_{x}$ and $I$ is indicated in (h). Arrows in (a) and (f) mark the deduced spin direction assuming a negligible perpendicular spin component.

spin character of the bulk states with enhanced intensity in agreement with the experimental observation.

Line profiles of intensity, spin polarization, and intensity of spin up/down electrons are depicted in Figs. 3(e)-3(g). The maximum spin polarization $P_{x}=0.2 \pm 0.05(0.1 \pm 0.05)$ of the outer (inner) bulk band is considerably smaller than that of the surface bands. A reduced in-plane spin polarization in combination with an out-of-plane component has been predicted in Ref. [12]. The physical origin of the difference of a factor of 2 in absolute polarization values for the inner and outer bulk Rashba band [Fig. 3(f)] indicates a spin-orbit induced hybridization between spin and multiple orbitals (instead of a single one) with nonzero orbital angular momenta, as has recently been proposed by Liu et al. [34] and confirmed experimentally by Maass et al. [21]. Deviating from the classical single-orbital model, the spin-orbit coupling 

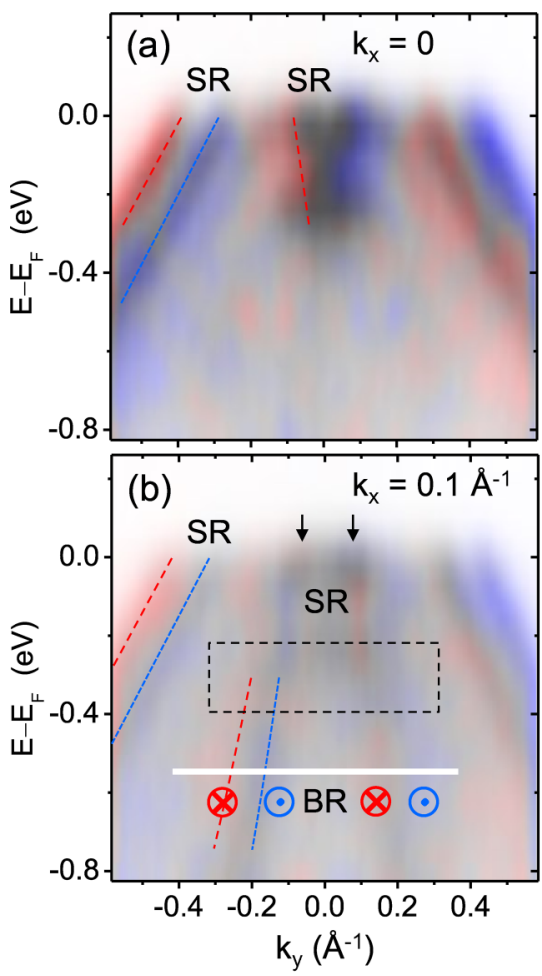
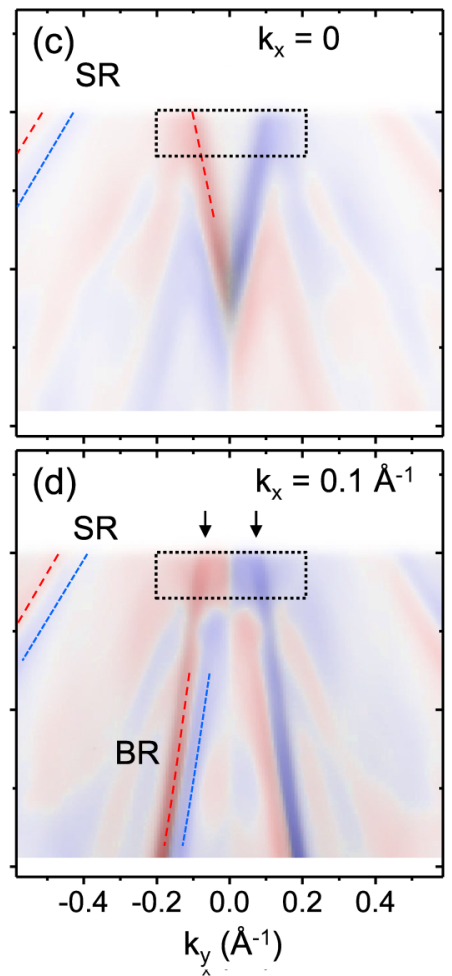
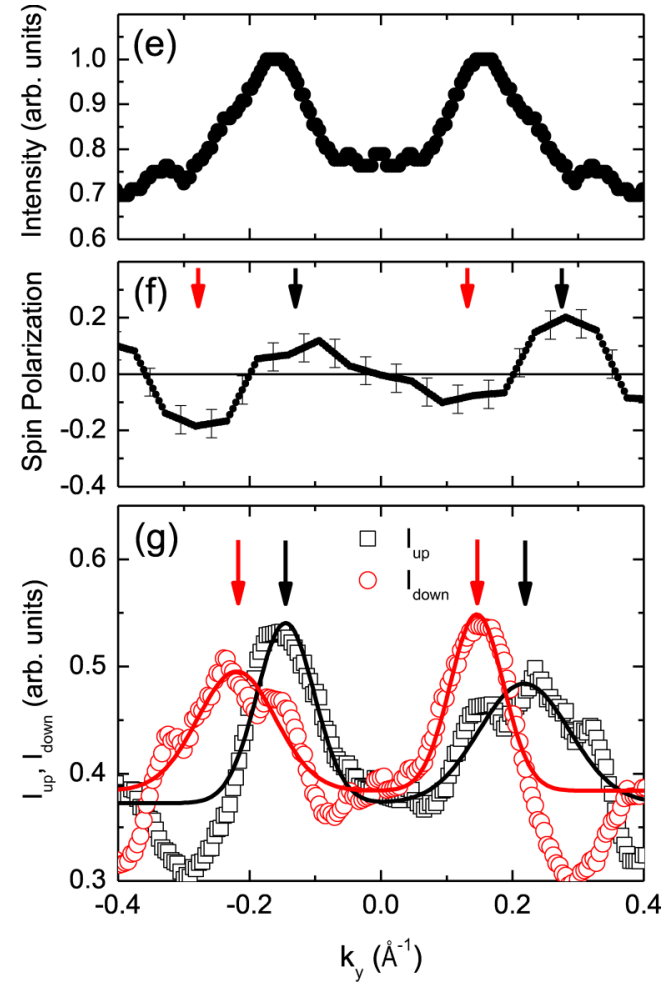

FIG. 3. (a), (b) Constant momentum cut $P_{x}\left(E_{b}, k_{x}, k_{y}\right)$ for $k_{x}=0$ and $k_{x}=+0.1 \AA^{-1}$ combined with the corresponding intensity distribution. For the color scale see Fig. 2. The cuts are averaged over a momentum interval of $\Delta k_{x}=0.05 \AA^{-1}$. Red (dashed) and blue (dotted) lines are guides to the eye; arrows and the dotted rectangle denote hybridization regions of bulk (BR) and surface states (SR). (c), (d) Calculations of the photoemission spin polarization and intensity shown in a similar representation as in (a) and (b): constant momentum cut $P_{x}\left(E_{b}, k_{x}, k_{y}\right)$ for $k_{x}=0(\mathrm{c})$ and $k_{x}=+0.1 \AA^{-1}$ (d). Red (dashed) and blue (dotted) lines are guides to the eye; arrows and dotted rectangle (d) denote the energy region of the surface resonance. (e) Intensity line profile $I\left(E_{b}, k_{x}, k_{y}\right)$ for $k_{x}=0.11 \AA^{-1}$ and $E_{b}=0.565 \mathrm{eV}$ along the line indicated in (b). (f) Corresponding line profile of the spin polarization $P_{x}$. $(\mathrm{g})$ Resulting intensity for spin-up and spin-down electrons. Arrows in (f) and (g) mark the extrema positions.

acts differently on each branch of the pair of spin-split bands. The in-plane $p$ orbitals couple to the same spin helicity, while the out-of-plane $p$ and $s$ orbitals couple to the opposite spin helicity [34]. Adding both contributions results in a net spin polarization for the band pair.

The partial overlap of the split bulk bands is responsible for the fact that the splitting appears larger in the spin profile than the theoretical Rashba splitting [see Fig. 3(d)]. A fit to the intensity profiles for spin-up and spin-down intensity [see Fig. 3(g)] results in a Rashba splitting of $\Delta k_{y}=0.07 \AA^{-1}$ in fair agreement with the theoretical value $\left(0.06 \AA^{-1}\right)$.

In summary, we experimentally evidenced a Rashbatype band splitting acting on bulk as opposed to surface electronic states in GeTe. The experimental results agree with fully relativistic density functional calculations of the photoemission intensities within the one-step model, confirming the relation of ground state and photoemission spin polarization. The spin polarization of the outer bulk Rashba branch is larger by a factor of 2 compared to the inner branch indicating a complex hybridization of bulk states. The net spin polarization may strongly affect the conversion process of spin into current via the spin-galvanic effect [34]. The spin splitting of bulk bands is caused by the inversion symmetry breaking of the crystal structure related to the electrical polarization as originally proposed in Ref. [5]. This rare bulk band splitting stands in contrast to the majority of previously investigated systems where the Rashba spin splitting is observed for surface states. The spin helicity is in line with the prediction for the equilibrium outward electrical polarization of $\mathrm{GeTe}(111)$ connecting it to the ferroelectricity of GeTe. The helicity is therefore switchable, thus representing the necessary precondition for technical applications of this material in nonvolatile spintronic devices.

The authors gratefully acknowledge financial support from the BMBF (Grants No. 05K13UM1, No. 05K13PA, No. 05K12EF1, and No. 05K13WMA), Deutsche Forschungsgemeinschaft (DFG EL172/18-1 and EB154/26-1 within SPP1666, FB 917-A3, and EB154/32-1), Center of Innovative Emerging Materials (CINEMA) and Stiftung Rheinland-Pfalz für Innovation (Project No. 1038) as well as CENTEM PLUS (LO1402).
[1] S. A. Wolf, D. D. Awschalom, R. A. Buhrman, J. M. Daughton, S. von Molnár, M. L. Roukes,
A. Y. Chtchelkanova, and D. M. Treger, Science 294, 1488 (2001). 
[2] I. Zutic, J. Fabian, and S. D. Sarma, Rev. Mod. Phys. 76, 323 (2004).

[3] Concepts in Spin Electronics, edited by S. Maekawa (Oxford University Press, Oxford, UK, 2006).

[4] D. D. Awschalom and M. E. Flatte, Nat. Phys. 3, 153 (2007).

[5] E. I. Rashba, Sov. Phys. Solid State 2, 1109 (1960).

[6] S. Datta and B. Das, Appl. Phys. Lett. 56, 665 (1990).

[7] J. Nitta, T. Akazaki, H. Takayanagi, and T. Enoki, Phys. Rev. Lett. 78, 1335 (1997).

[8] G. Engels, J. Lange, T. Schäpers, and H. Lüth, Phys. Rev. B 55, R1958(R) (1997).

[9] H. C. Koo, J. H. Kwon, J. Eom, J. Chang, S. H. Han, and M. Johnson, Science 325, 1515 (2009).

[10] J. Wunderlich, B. G. Park, A. C. Irvine, L. P. Zarbo, E. Rozkotova, P. Nemec, V. Novak, J. Sinova, and T. Jungwirth, Science 330, 1801 (2010).

[11] C. Betthausen, T. Dollinger, H. Saarikoski, V. Kolkovsky, G. Karczewski, T. Wojtowicz, K. Richter, and D. Weiss, Science 337, 324 (2012).

[12] D. D. Sante, P. Barone, R. Bertacco, and S. Picozzi, Adv. Mater. 25, 509 (2013).

[13] M. Liebmann, C. Rinaldi, D. D. Sante, J. Kellner, C. Pauly, R. N. Wang, J. E. Boschker, A. Giussani, S. Bertoli, M. Cantoni, L. Baldrati, I. Vobornik, G. Panaccione, D. Marchenko, J. Sanchez-Barriga, O. Rader, R. Calarco, S. Picozzi, R. Bertacco, and M. Morgenstern, Adv. Mater. 28, 560 (2016).

[14] J. Krempaský, H. Volfová, S. Muff, N. Pilet, G. Landolt, M. Radović, M. Shi, D. Kriegner, V. Holý, J. Braun, H. Ebert, F. Bisti, V. A. Rogalev, V. N. Strocov, G. Springholz, J. Minár, and J. H. Dil, arXiv:1503.05004.

[15] G. Dresselhaus, Phys. Rev. 100, 580 (1955).

[16] X. Zhang, Q. Liu, J. W. Luo, A. J. Freeman, and A. Zunger, Nat. Phys. 10, 387 (2014).

[17] K. Ishizaka et al., Nat. Mater. 10, 521 (2011).

[18] J. S. Lee, G. A. H. Schober, M. S. Bahramy, H. Murakawa, Y. Onose, R. Arita, N. Nagaosa, and Y. Tokura, Phys. Rev. Lett. 107, 117401 (2011).

[19] C.-R.Wang, J.-C. Tung, R. Sankar, C.-T. Hsieh, Y.-Y. Chien, G. Y. Guo, F. C. Chou, and W.-L. Lee, Phys. Rev. B 88, 081104 (2013).

[20] M. Sakano, M. S. Bahramy, A. Katayama, T. Shimojima, H. Murakawa, Y. Kaneko, W. Malaeb, S. Shin, K. Ono, H.
Kumigashira, R. Arita, N. Nagaosa, H. Y. Hwang, Y. Tokura, and K. Ishizaka, Phys. Rev. Lett. 110, 107204 (2013).

[21] H. Maass, H. Bentmann, C. Seibel, C. Tusche, S. V. Eremeev, T. Peixoto, O. E. Tereshchenko, K. Kokh, E. C. J. Kirschner, and F. Reinert, Nat. Commun. 7, 11621 (2016).

[22] A. V. Kolobov, D. J. Kim, A. Giussani, P. Fons, J. Tominaga, R. Calarco, and A. Gruverman, APL Materials 2, 066101 (2014).

[23] C. Rinaldi, J. C. Rojas-Sánchez, R. N. Wang, Y. Fu, S. Oyarzun, L. Vila, S. Bertoli, M. Asa, L. Baldrati, M. Cantoni, J.-M. George, R. Calarco, A. Fert, and R. Bertacco, APL Materials 4, 032501 (2016).

[24] G. Schönhense, K. Medjanik, and H. Elmers, J. Electron Spectrosc. Relat. Phenom. 200, 94 (2015).

[25] M. Kolbe, P. Lushchyk, B. Petereit, H. J. Elmers, G. Schönhense, A. Oelsner, C. Tusche, and J. Kirschner, Phys. Rev. Lett. 107, 207601 (2011).

[26] F. Ji, T. Shi, M. Ye, W. Wan, Z. Liu, J. Wang, T. Xu, and S. Qiao, Phys. Rev. Lett. 116, 177601 (2016).

[27] R. Wang, J. Boschker, E. Bruyer, D. D. Sante, S. Picozzi, K. Perumal, A. Giussani, H. Riechert, and R. Calarco, J. Phys. Chem. C 118, 29724 (2014).

[28] D. Kutnyakhov, H. J. Elmers, G. Schönhense, C. Tusche, S. Borek, J. Braun, J. Minar, and H. Ebert, Phys. Rev. B 91, 014416 (2015)

[29] C. Tusche, M. Ellguth, A. Krasyuk, A. Winkelmann, D. Kutnyakhov, P. Lushchyk, K. Medjanik, G. Schönhense, and J. Kirschner, Ultramicroscopy 130, 70 (2013).

[30] H. Ebert, D. Ködderitzsch, and J. Minár, Rep. Prog. Phys. 74, 096501 (2011).

[31] H. Ebert, J. Braun, and J. Minar, The Munich SPRKKR package, version 6.3, http://olymp.cup.uni-muenchen.de/ ak/ebert/SPRKKR.

[32] J. Braun, K. Miyamoto, A. Kimura, T. Okuda, M. Donath, H. Ebert, and J. Minar, New J. Phys. 16, 015005 (2014).

[33] See Supplemental Material at http://link.aps.org/supplemental/ 10.1103/PhysRevB.94.201403 for a detailed comparison of experimental and theoretically expected spin texture of the surface Rashba band.

[34] Q. Liu, X. Zhang, J. A. Waugh, D. S. Dessau, and A. Zunger Phys. Rev. B 94, 125207 (2016). 